BONN-TH-97-05

hep-ph/9703368

\title{
The Gerasimov-Drell-Hearn sum rule and the infinite-momentum limit
}

\author{
Ralf Pantförder円, Horst Rollnik, and Walter Pfeil \\ Physikalisches Institut der Universität Bonn \\ Nußallee 12, 53115 Bonn, Germany
}

\begin{abstract}
We study the current-algebra approach to the Gerasimov-Drell-Hearn sum rule, paying particular attention to the infinite-momentum limit. Employing the $\mathcal{O}\left(\alpha^{2}\right)$ WeinbergSalam model of weak interactions as a testing ground, we find that the legitimacy of the infinite-momentum limit is intimately connected with the validity of the naive equal-times algebra of electric charge densities. Our results considerably reduce the reliability of a recently proposed modification of the Gerasimov-Drell-Hearn sum rule, originating from an anomalous charge-density algebra.
\end{abstract}

1e-mail: pantfoer@thew01.physik.uni-bonn.de 


\section{Introduction}

Exciting results on the spin structure of the proton derived from deep inelastic scattering experiments with polarized muons and protons brought the attention of particle physicists back to a rather old issue: by general arguments of field theory and particle theory there is a relation between a particle's anomalous magnetic moment and an integral of a particular combination of the spin-dependent photoabsorption cross sections of that particle.

For the nucleon, this relation is given by the Gerasimov-Drell-Hearn (GDH) sum rule [1, 2], a direct experimental test of which being in progress at several accelerators. Yet there are estimates taken in an indirect fashion from pion-photoproduction data [3, 4, 5, 6, 7]. Since the integral in question runs over all photon energies, one has to extrapolate the data, and even certain model assumptions about multi-particle final states have to be made. Nevertheless, a definite discrepancy, particularly for the proton-neutron difference, remains outside reasonable uncertainties, thus leading to a basic problem for photo-hadron physics.

In view of this situation, various proposals to alter the GDH sum rule have been published [8, 9, 10]. In this article we point to a general difficulty which all of these attempts encounter.

The GDH sum rule was originally derived from dispersion theory [1, 2]. Conventional Regge phenomenology predicts the dispersion integral to converge [11] (see also Ref. 12]), which has to do with moving Regge poles only. However, as Abarbanel and Goldberger [13] pointed out, a possible fixed pole at angular momentum $J=1$ would modify the sum rule by an additive constant - essentially the residue of the fixed pole. We stress that it is by no means evident that the fixed pole should be absent. On the other hand, there is as yet no reliable model prediction for the magnitude of its residue.

Alternatively, by the current-algebra approach, the GDH sum rule was founded on the completeness sum in the infinite-momentum limit [14, 15], assuming that the operators of electric charge densities commute at equal times. A few attempts to go beyond this assumption can be found in the literature $[8,9,10$. However, these investigations suffer from a severe deficiency which has not been noted before: the infinite-momentum limit is handled in a naive way, the legitimacy of which cannot be based on current algebra alone - it enters as a mere conjecture.

In the present article, we analyze the significance of the problems connected with the infinitemomentum limit in some detail. To this end, we firstly derive the form that the GDH sum rule gets without taking the infinite-momentum limit. We call this equation the finite-momentum GDH sum rule. It is formulated in terms of the (timelike) virtual forward Compton amplitude of the nucleon, or any other fermion under consideration. To examine the legitimacy of the infinite-momentum limit, we need a perturbative model that allows us to calculate the Compton amplitude for all values of the photon's energy and virtuality. Unfortunately, for the nucleon there is no realistic model that works in the relevant kinematical domain. Therefore, following Ref. [16], we take the external fermion to be an electron and employ the $\mathcal{O}\left(\alpha^{2}\right)$ Weinberg-Salam model, i.e. the standard model of electroweak interactions of leptons to the fourth order of perturbation theory. To this order, tree-level and one-loop Feynman graphs involving $\mathrm{e}^{-}, \nu_{\mathrm{e}}, \gamma, \mathrm{W}^{ \pm}, \mathrm{Z}^{0}$, and Higgs as internal particles are to be worked out.

We obtain a non-vanishing charge-density commutator due to the presence of the triangle anomaly, giving rise to a modification of the finite-momentum GDH sum rule. Our key result, however, is that if the infinite-momentum limit is handled with care, it brings about a second modification which exactly cancels the previous one, hence leaving the (infinite-momentum) GDH sum rule unaltered.

We are thus led to the conclusion that the infinite-momentum limit has to be regarded as being just as critical as the charge-density commutator, especially in the presence of anomalous commutators. This fact greatly reduces the reliability of any proposed modification of the GDH sum rule.

After some preliminaries in section 2, we review in section 3 the dispersion-theoretic approach to the GDH sum rule. Section 4 gives the derivation of the finite-momentum GDH sum rule. Section 5 introduces the $\mathcal{O}\left(\alpha^{2}\right)$ Weinberg-Salam model and presents the modifications brought about by charge-density algebra and infinite-momentum limit. 


\section{Preliminaries}

Throughout this paper, $M, Z$, and $\kappa$ denote the nucleon's mass, charge (in units of $e$ ), and anomalous magnetic moment (in units of $\mu_{\mathrm{N}}$ ), respectively, and $\alpha=e^{2} / 4 \pi$ is the fine-structure constant. One-nucleon states $|p, \lambda\rangle$ with four-momentum $p$ and helicity $\lambda= \pm 1 / 2$ are normalized covariantly, $\left\langle p, \lambda \mid p^{\prime}, \lambda^{\prime}\right\rangle=(2 \pi)^{3} 2 p^{0} \delta^{3}\left(\boldsymbol{p}-\boldsymbol{p}^{\prime}\right) \delta_{\lambda \lambda^{\prime}}$. Spinors $u(p, \lambda)$ are normalized as $\bar{u}(p, \lambda) u(p, \lambda)=2 M \delta_{\lambda \lambda^{\prime}}$. Besides this helicity basis, we use states $|p, s\rangle$ and spinors $u(p, s)$ with arbitrary spin four-vector $s$ obeying $s^{2}=-1, p \cdot s=0$, and $\bar{u}(p, s) \gamma^{\mu} \gamma_{5} u(p, s)=2 M s^{\mu}$. Helicity eigenstates are those for which the three-vectors $\boldsymbol{p}$ and $s$ are collinear. For details we refer to the textbooks, e.g. Ref. [17, section 2-2]. We adopt the conventions $\epsilon^{123}=\epsilon^{0123}=+1$ and $\gamma_{5}=i \gamma^{0} \gamma^{1} \gamma^{2} \gamma^{3}$.

We consider the virtual forward Compton amplitude

$$
T^{\mu \nu}(p, q, s)=i \int \mathrm{d}^{4} x e^{i q \cdot x}\left\langle p, s\left|\mathrm{~T} J^{\mu}(x) J^{\nu}(0)\right| p, s\right\rangle .
$$

Its antisymmetric part has the invariant decomposition

$$
\frac{1}{2}\left(T^{\mu \nu}-T^{\nu \mu}\right)=-\frac{i}{M} \epsilon^{\mu \nu \rho \sigma} q_{\rho} s_{\sigma} A_{1}\left(\nu, q^{2}\right)-\frac{i}{M^{3}} \epsilon^{\mu \nu \rho \sigma} q_{\rho}\left((M \nu) s_{\sigma}-(q \cdot s) p_{\sigma}\right) A_{2}\left(\nu, q^{2}\right),
$$

where $\nu=p \cdot q / M$ is the lab-frame energy of the photon. In the current-algebra approach to the GDH sum rule, the linear combination

$$
f_{2}\left(\nu, q^{2}\right)=\frac{\alpha}{2 M^{2}}\left(A_{1}\left(\nu, q^{2}\right)+\frac{q^{2}}{M \nu} A_{2}\left(\nu, q^{2}\right)\right)
$$

of the dimensionless invariant amplitudes $A_{1,2}\left(\nu, q^{2}\right)$ emerges naturally. For real photons we define as usual $f_{2}(\nu) \equiv f_{2}(\nu, 0)$.

\section{Dispersion-theoretic approach to the GDH sum rule}

We now remind to the original dispersion-theoretic approach of refs. [1, 2], which assumes an unsubtracted dispersion relation for the forward Compton amplitude $f_{2}(\nu)$,

$$
\operatorname{Re} f_{2}(\nu)=\frac{2}{\pi} \mathcal{P} \int_{\nu_{0}}^{\infty} \frac{\nu^{\prime} \mathrm{d} \nu^{\prime}}{\nu^{\prime 2}-\nu^{2}} \operatorname{Im} f_{2}\left(\nu^{\prime}\right) .
$$

The constant $\nu_{0}=m_{\pi}+m_{\pi}^{2} / 2 M$ is the pion-photoproduction threshold, and $\mathcal{P}$ denotes principal value integration, which can be omitted in case $\nu<\nu_{0}$. Taking $\nu=0$ in Eq. (3.1) yields

$$
f_{2}(0)=\frac{2}{\pi} \int_{\nu_{0}}^{\infty} \frac{\mathrm{d} \nu^{\prime}}{\nu^{\prime}} \operatorname{Im} f_{2}\left(\nu^{\prime}\right) .
$$

The GDH sum rule

$$
-\frac{2 \pi^{2} \alpha \kappa^{2}}{M^{2}}=\int_{\nu_{0}}^{\infty} \frac{\mathrm{d} \nu}{\nu}\left(\sigma_{1 / 2}(\nu)-\sigma_{3 / 2}(\nu)\right)
$$

is obtained from Eq. (3.2) by using the low-energy theorem of Low [18] and Gell-Mann and Goldberger [19],

$$
f_{2}(0)=-\frac{\alpha \kappa^{2}}{2 M^{2}}
$$

and the optical theorem for the imaginary part of the forward amplitude,

$$
8 \pi \operatorname{Im} f_{2}(\nu)=\sigma_{1 / 2}(\nu)-\sigma_{3 / 2}(\nu) .
$$


Here, $\sigma_{1 / 2}(\nu)$ and $\sigma_{3 / 2}(\nu)$ denote the photoabsorption cross sections of the nucleon for total photon-nucleon helicities $1 / 2$ and $3 / 2$, respectively.

The validity of the unsubtracted dispersion relation (3.1) requires not only the imaginary part of $f_{2}(\nu)$ to vanish sufficiently rapid at large $\nu$ in order that the integral will converge. Besides, the real part has to vanish too, which means $f_{2}(\infty)=0$. That this need not necessarily be the case was shown by Abarbanel and Goldberger [13. In Regge language, a possible non-vanishing $f_{2}(\infty)$ is equivalent to a $J=1$ fixed pole in angular-momentum plane.

The easiest way to see what modification is brought about by a non-vanishing $f_{2}(\infty)$, is to write down a subtracted dispersion relation,

$$
\operatorname{Re} f_{2}(\nu)-f_{2}(0)=\frac{2}{\pi} \mathcal{P} \int_{\nu_{0}}^{\infty} \frac{\nu^{2} \mathrm{~d} \nu^{\prime}}{\nu^{\prime}\left(\nu^{\prime 2}-\nu^{2}\right)} \operatorname{Im} f_{2}\left(\nu^{\prime}\right) .
$$

Letting $\nu$ approach infinity now, one gets

$$
f_{2}(0)=\frac{2}{\pi} \int_{\nu_{0}}^{\infty} \frac{\mathrm{d} \nu^{\prime}}{\nu^{\prime}} \operatorname{Im} f_{2}\left(\nu^{\prime}\right)+f_{2}(\infty)
$$

This gives rise to a finite modification of the GDH sum rule. Note that the subtraction here was not enforced by a divergent integral, in which case it would have been impossible to drag the limit $\nu \rightarrow \infty$ inside the $\nu^{\prime}$ integral in Eq. (3.6).

We emphasize that to our knowledge, there is no fundamental reason requesting the constant $f_{2}(\infty)$ to vanish!

\section{Current-algebra approach - the finite-momentum GDH sum rule}

In this section we remind the reader to the current-algebra derivation of the GDH sum rule, which is based essentially on two premises. Firstly, electric charge densities are assumed to commute at equal times. Secondly, one assumes that taking the infinite-momentum limit is legitimate. We stress that there are ansätze [8, 9, 10] that weaken the former assumption, but the latter one has never been questioned seriously.

We follow the idea of Hosoda and Yamamoto [14], but we postpone the infinite-momentum limit to the very end of the calculation in order to shed some light on its meaning. For the sake of transparency, we explicitely write down some formulae which are known from the literature, e.g. Ref. 17].

From causality arguments, the equal-times commutator $\left[J^{0}(\boldsymbol{x}, 0), J^{0}(\boldsymbol{y}, 0)\right]$ of electric charge densities must be a finite sum over derivatives of the delta function $\delta^{3}(\boldsymbol{x}-\boldsymbol{y})$. Here we start from the naive commutator

$$
\left[J^{0}(\boldsymbol{x}, 0), J^{0}(\boldsymbol{y}, 0)\right]=0,
$$

which can formally be obtained by writing $J^{0}(x)=\sum_{\mathrm{f}} Z_{\mathrm{f}} q_{\mathrm{f}}^{\dagger}(x) q_{\mathrm{f}}(x)$ and employing canonical anticommutation relations among quark fields $q_{\mathrm{f}}^{\dagger}(x), q_{\mathrm{f}}(x)$. We define the operator of the electric dipole moment as usual,

$$
D^{i}\left(x^{0}\right)=e \int \mathrm{d}^{3} x x^{i} J^{0}(x)
$$

and sandwich the commutator of components $D^{ \pm}(0) \equiv\left(D^{1}(0) \pm i D^{2}(0)\right) / \sqrt{2}$ between one-nucleon states of positive helicity, taking the incoming nucleon to be traveling along the $x^{3}$-axis, $p^{\mu}=$ $\left(p^{0}, 0,0, \sqrt{\left(p^{0}\right)^{2}-M^{2}}\right)$,

$$
\left\langle p^{\prime}, \frac{1}{2}\left|\left[D^{+}(0), D^{-}(0)\right]\right| p, \frac{1}{2}\right\rangle=0 .
$$

We now insert a complete set of intermediate states and separate the one-nucleon states from the continuum. 
In terms of form factors $F_{1,2}\left(q^{2}\right)$ with normalization $F_{1}(0)=Z, F_{2}(0)=\kappa$, the one-nucleon matrix element of the dipole-moment operator reads

$$
\left\langle k, \lambda\left|D^{i}(0)\right| p, \frac{1}{2}\right\rangle=i e(2 \pi)^{3} \nabla^{i} \delta^{3}(\boldsymbol{q}) u^{\dagger}(k, \lambda)\left(F_{1}\left(q^{2}\right)+\gamma \cdot \boldsymbol{q} \frac{F_{2}\left(q^{2}\right)}{2 M}\right) u\left(p, \frac{1}{2}\right),
$$

where $q=k-p$. Therewith, the one-nucleon intermediate-state contribution to the matrix element (4.3) is obtained straightforwardly,

$$
\begin{aligned}
\left\langle p^{\prime}, \frac{1}{2}\left|\left[D^{+}(0), D^{-}(0)\right]\right| p, \frac{1}{2}\right\rangle_{\text {one-nucleon }}= & \sum_{\lambda= \pm \frac{1}{2}} \int \frac{\mathrm{d}^{3} k}{(2 \pi)^{3} 2 k^{0}}\left\langle p^{\prime}, \frac{1}{2}\left|D^{+}(0)\right| k, \lambda\right\rangle\left\langle k, \lambda\left|D^{-}(0)\right| p, \frac{1}{2}\right\rangle \\
& -\{+\leftrightarrow-\} \\
= & (2 \pi)^{3} 2 p^{0} \delta^{3}\left(\boldsymbol{p}^{\prime}-\boldsymbol{p}\right)\left(\frac{2 \pi \alpha \kappa^{2}}{M^{2}}-\frac{2 \pi \alpha(Z+\kappa)^{2}}{\left(p^{0}\right)^{2}}\right) .
\end{aligned}
$$

Here we stress the presence of the second term, which vanishes in the infinite-momentum limit. Hitherto, its only appearance in the literature was in Ref. [20], where it was incorrect.

On the other hand, the continuum contribution, i.e. the sum over all intermediate states $|\mathrm{I}\rangle$ except the one-nucleon state, can be obtained by virtue of current conservation $\partial_{\mu} J^{\mu}(x)=0$, which implies $\dot{D}^{i}\left(x^{0}\right)=e \int \mathrm{d}^{3} x J^{i}(x)$, and using translational invariance to carry out the spatial integrations,

$$
\begin{aligned}
\left\langle p^{\prime}, \frac{1}{2}\left|\left[D^{+}(0), D^{-}(0)\right]\right| p, \frac{1}{2}\right\rangle_{\mathrm{cont}}= & \sum_{\mathrm{I}}^{\prime}\left\langle p^{\prime}, \frac{1}{2}\left|D^{+}(0)\right| \mathrm{I}\right\rangle\left\langle\mathrm{I}\left|D^{-}(0)\right| p, \frac{1}{2}\right\rangle-\{+\leftrightarrow-\} \\
= & (2 \pi)^{3} \delta^{3}\left(\boldsymbol{p}^{\prime}-\boldsymbol{p}\right) \sum_{\mathrm{I}}^{\prime}(2 \pi)^{3} \delta^{3}\left(\boldsymbol{p}_{\mathrm{I}}-\boldsymbol{p}\right) \frac{4 \pi \alpha\left|\left\langle p, \frac{1}{2}\left|J^{+}(0)\right| \mathrm{I}\right\rangle\right|^{2}}{\left(p^{0}-p_{\mathrm{I}}^{0}\right)^{2}} \\
& -\{+\rightarrow-\} .
\end{aligned}
$$

The \pm components of the current are defined by $J^{ \pm}(x) \equiv\left(J^{1}(x) \pm i J^{2}(x)\right) / \sqrt{2}$. Introducing the timelike virtual photon momentum $q$ with $\boldsymbol{q}=0$, we can substitute $\delta^{3}\left(\boldsymbol{p}_{\mathrm{I}}-\boldsymbol{p}\right)=\int_{q_{\mathrm{thr}}^{0}}^{\infty} \mathrm{d} q^{0} \delta^{4}\left(p_{\mathrm{I}}-p-\right.$ $q$ ), where the pion-production threshold $q_{\mathrm{thr}}^{0} \equiv M \nu_{\mathrm{thr}} / p^{0}$ is determined by $\left(p+q_{\mathrm{thr}}\right)^{2}=\left(M+m_{\pi}\right)^{2}$. For $p^{0} \rightarrow \infty, \nu_{\text {thr }}$ approaches the familiar pion-photoproduction threshold $\nu_{0}=m_{\pi}+m_{\pi}^{2} / 2 M$.

We can now express the continuum contribution in terms of the forward virtual Compton amplitude $f_{2}\left(\nu, q^{2}\right)$, Eq. (2.3),

$$
\begin{aligned}
\left\langle p^{\prime}, \frac{1}{2}\left|\left[D^{+}(0), D^{-}(0)\right]\right| p, \frac{1}{2}\right\rangle_{\text {cont }}= & (2 \pi)^{3} 2 p^{0} \delta^{3}\left(\boldsymbol{p}^{\prime}-\boldsymbol{p}\right) \int_{q_{\mathrm{thr}}^{0}}^{\infty} \frac{\mathrm{d} q^{0}}{q^{0}} \frac{\alpha}{p^{0} q^{0}} \int \mathrm{d}^{4} x e^{i q x}\left\langle p, \frac{1}{2}\left|J^{+}(x) J^{-}(0)\right| p, \frac{1}{2}\right\rangle \\
& -\{+\leftrightarrow-\} \\
= & (2 \pi)^{3} 2 p^{0} \delta^{3}\left(\boldsymbol{p}^{\prime}-\boldsymbol{p}\right) 8 \int_{\nu_{\mathrm{thr}}}^{\infty} \frac{\mathrm{d} \nu}{\nu} \operatorname{Im} f_{2}\left(\nu, \frac{M^{2} \nu^{2}}{\left(p_{0}\right)^{2}}\right)
\end{aligned}
$$

Since the one-nucleon part (4.5) and the continuum part (4.7) sum up to give the commutator matrix element (4.3), we conclude

$$
-\frac{2 \pi^{2} \alpha \kappa^{2}}{M^{2}}+\frac{2 \pi^{2} \alpha(Z+\kappa)^{2}}{\left(p^{0}\right)^{2}}=\int_{\nu_{\mathrm{thr}}}^{\infty} \frac{\mathrm{d} \nu}{\nu} 8 \pi \operatorname{Im} f_{2}\left(\nu, \frac{M^{2} \nu^{2}}{\left(p^{0}\right)^{2}}\right) .
$$

We call this equation the finite-momentum GDH sum rule. It is based solely on the naive chargedensity commutator (4.1), or on the weaker assumption presented by Eq. (4.3). In particular, the integral on the right-hand side of Eq. (4.8) converges, since it relies only on the validity of the completeness relation for the physical intermediate states. This is irrespective of the convergence of the genuine GDH integral with its integrand $8 \pi \operatorname{Im} f_{2}(\nu, 0) / \nu$. The integration path in the $\left(\nu, q^{2}\right)$ plane for various values of the energy $p^{0}$ is depicted in Fig. 1. Note that for any finite value of $p^{0}$ this path is a parabola that extends to arbitrarily high timelike photon virtualities. 


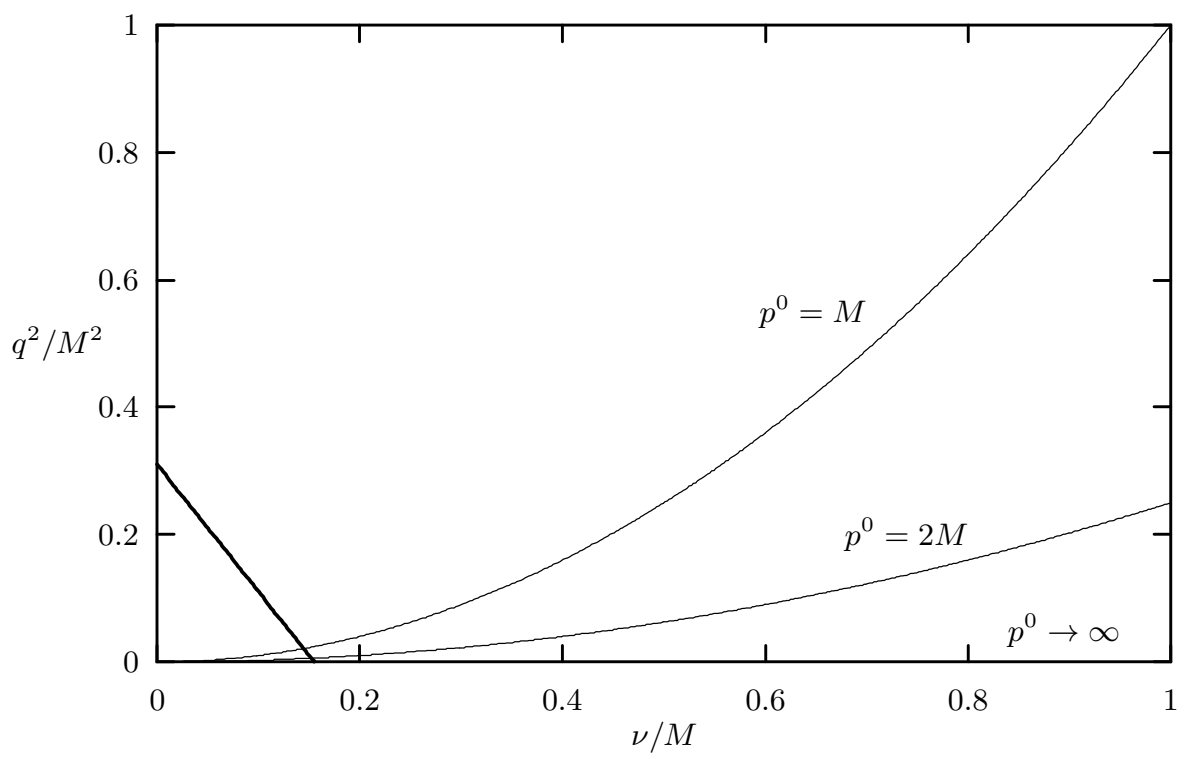

Figure 1: The integration path of the finite-momentum GDH sum rule $(4.8)$ in the $\left(\nu, q^{2}\right)$ plane for nucleon energy $p^{0}=M$ and $p^{0}=2 M$. The heavy line represents the pion-production threshold.

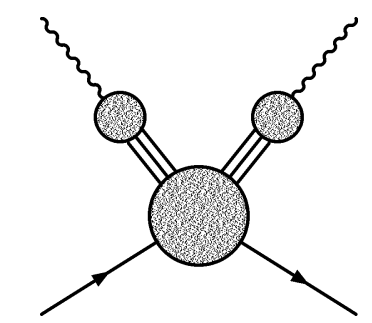

Figure 2: Intermediate hadron states in virtual Compton scattering, leading to singularities in the photon mass $q^{2}$.

Taking the infinite-momentum limit now constitutes the last but one step of the derivation of the GDH sum rule,

$$
-\frac{2 \pi^{2} \alpha \kappa^{2}}{M^{2}}=\lim _{p^{0} \rightarrow \infty} \int_{\nu_{\mathrm{thr}}}^{\infty} \frac{\mathrm{d} \nu}{\nu} 8 \pi \operatorname{Im} f_{2}\left(\nu, \frac{M^{2} \nu^{2}}{\left(p^{0}\right)^{2}}\right) .
$$

To get the usual form of the sum rule one now has to interchange the limit $p^{0} \rightarrow \infty$ with the $\nu$ integration. If the properties of the function $\operatorname{Im} f_{2}\left(\nu, q^{2}\right)$ allow the limit to be dragged into the integral, the GDH sum rule follows from Eq. (4.9) with the help of the optical theorem (3.5). In principle, however, permuting limit and integration could give rise to a (finite or infinite) modification of the sum rule. We stress that current algebra has no answer to this problem.

Nevertheless, one can easily see the origin of possible difficulties on quite general grounds. We recall that the timelike virtual Compton amplitude meets singularities in the photon mass $q^{2}$ due to intermediate hadron states, as indicated in Fig. 2. Thus one expects for the amplitude $f_{2}\left(\nu, q^{2}\right)$ a spectral representation of the form

$$
\operatorname{Im} f_{2}\left(\nu, q^{2}\right)=\frac{1}{\pi} \int_{q_{0}^{2}}^{\infty} \frac{\mathrm{d} q^{\prime 2}}{q^{2}-q^{\prime 2}} \rho\left(\nu, q^{\prime 2}\right)
$$

where $q_{0}^{2}$ is the mass of the lowest-lying state that couples to the photon. Inserting Eq. (4.10) into Eq. (4.9), it is evident that for finite $p^{0}$, the $\nu$ integration also meets the $q^{2}$ singularities, 
since $q^{2}=M^{2} \nu^{2} /\left(p^{0}\right)^{2}$. Only if one can drag the limit $p^{0} \rightarrow \infty$ inside the integral in Eq. (4.9), these singularities play no explicite role.

In the following section we will show, by adopting a concrete model, that indeed non-trivial modifications of the current-algebra arguments are to be expected.

\section{The finite-momentum GDH sum rule within the $\mathcal{O}\left(\alpha^{2}\right)$ Weinberg-Salam model}

We now examine the finite-momentum GDH sum rule and the infinite-momentum limit for the Compton amplitude of the electron within the $\mathcal{O}\left(\alpha^{2}\right)$ Weinberg-Salam model. Let $m$ and $M_{\mathrm{Z}}$ denote the mass of the electron and the $\mathrm{Z}^{0}$ boson, respectively. For simplicity, we will take the Weinberg angle $\theta_{\mathrm{W}}$ to be such that the coupling of the $\mathrm{Z}^{0}$ boson to electrons is purely axial-vector, i.e. $\sin ^{2} \theta_{\mathrm{W}}=1 / 4$. The Fermi constant is then given by $G_{\mathrm{F}} / \sqrt{2}=8 \pi \alpha / 3 M_{\mathrm{Z}}^{2}$. We will expand in the coupling constant $e$ only, regarding all masses as given parameters, thus $m^{2} G_{\mathrm{F}}$ will be of order $\alpha$.

In 1972, Altarelli, Cabibbo, and Maiani [16] investigated the GDH sum rule for the $\mathcal{O}\left(\alpha^{2}\right)$ Weinberg-Salam model by calculating the forward amplitude $f_{2}(\nu)$ for the real Compton process and checking explicitely that it obeys an unsubtracted dispersion relation. The Feynman graphs up to order $\alpha^{2}$ are presented in Fig. 3. The tree graphs (a), as well as the contact graph (e) and the Higgs-exchange graphs (f), do not contribute to the antisymmetric piece of the Compton amplitude $T^{\mu \nu}$, thus having no effect on $f_{2}(\nu)$. As will be demonstrated below, in the case of a real photon also the $Z^{0}$-exchange graphs of Fig. 3(g) vanish. In view of the fact that the anomalous magnetic moment $\kappa$ of the electron is of order $\alpha$, the left-hand side of the GDH sum rule (3.3) is of order $\alpha^{3}$, so that to order $\alpha^{2}$ it reads

$$
0=\int_{0}^{\infty} \frac{\mathrm{d} \nu}{\nu} \operatorname{Im} f_{2}(\nu) .
$$

This relation is proven in Ref. [16] (see also Ref. [21]). Since $\mathrm{d} \nu / \nu=\mathrm{d}(\ln \nu)$, Eq. (5.1) is reflected by the equality of the shaded areas in Fig. A, which shows the QED contribution to the function $8 \pi \operatorname{Im} f_{2}(\nu)=\sigma_{1 / 2}(\nu)-\sigma_{3 / 2}(\nu)$, i.e. the contribution due to the $\mathrm{e}^{-} \gamma$ intermediate states of Fig. 3(b).

Here we address the question whether the current-algebra approach to the GDH sum rule also works within the $\mathcal{O}\left(\alpha^{2}\right)$ Weinberg-Salam model. To be precise, we will investigate the validity of the naive dipole-moment commutator (4.3), and the legitimacy of the infinite-momentum limit. We will find that both assumptions are violated, due to the same Feynman graphs, namely the $\mathrm{Z}^{0}$-exchange graphs of Fig. 3(f). There is a modification of the finite-momentum GDH sum rule, but this modification is removed when the infinite-momentum limit is taken, leading back to the original GDH sum rule (5.1).

We adopt the Bjorken-Johnson-Low (BJL) technique [22, 23] to work out the one-electron matrix element of the charge-density commutator. The BJL limit

$$
\int \mathrm{d}^{3} x e^{-i \boldsymbol{q}^{\prime} \cdot \boldsymbol{x}}\left\langle p^{\prime}, \frac{1}{2}\left|\left[J^{0}(\boldsymbol{x}, 0), J^{0}(0)\right]\right| p, \frac{1}{2}\right\rangle=-\lim _{q^{\prime 0} \rightarrow \infty} q^{\prime 0} T^{00}\left(p, p^{\prime}, q^{\prime}\right)
$$

relates the commutator matrix element to the (generally non-forward) virtual Compton amplitude

$$
e^{2} T^{\mu \nu}\left(p, p^{\prime}, q^{\prime}\right)=i e^{2} \int \mathrm{d}^{4} x e^{i q^{\prime} \cdot x}\left\langle p^{\prime}, \frac{1}{2}\left|\mathrm{~T} J^{\mu}(x) J^{\nu}(0)\right| p, \frac{1}{2}\right\rangle
$$

which we study perturbatively. All polynomials in $q^{\prime 0}$, the so-called seagulls, have to be dropped in this procedure. A typical seagull is presented by the Feynman graph of Fig. 3(e), which is completely independent of the photon momenta $q$ and $q^{\prime}$.

We found that to order $\alpha^{2}$, only the $\mathrm{Z}^{0}$-exchange graphs of Fig. $3(\mathrm{f})$ contribute. This may not be surprising, since these fermion triangle graphs are responsible for the famous Adler-Bell-Jackiw anomaly [24, 25]. Details of our calculation are given in the appendix. The result for the matrix element of the dipole-moment commutator is non-vanishing,

$$
\left\langle p^{\prime}, \frac{1}{2}\left|\left[D^{+}(0), D^{-}(0)\right]\right| p, \frac{1}{2}\right\rangle=(2 \pi)^{3} 2 p^{0} \delta^{3}\left(\boldsymbol{p}^{\prime}-\boldsymbol{p}\right) \frac{\alpha}{\pi} \frac{G_{\mathrm{F}}}{\sqrt{2}},
$$


(a)

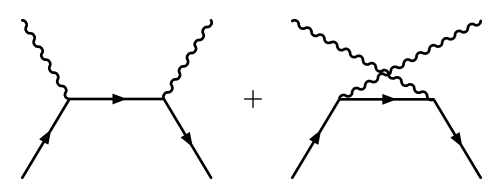

(b)

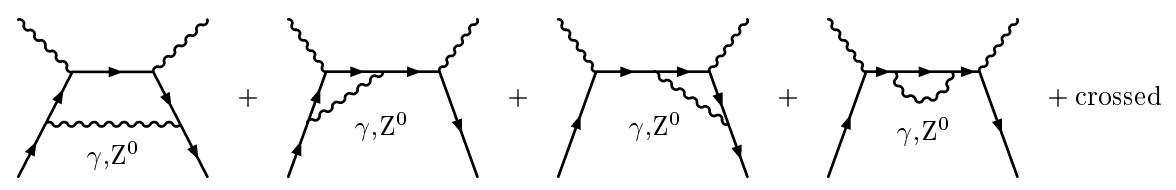

(c)

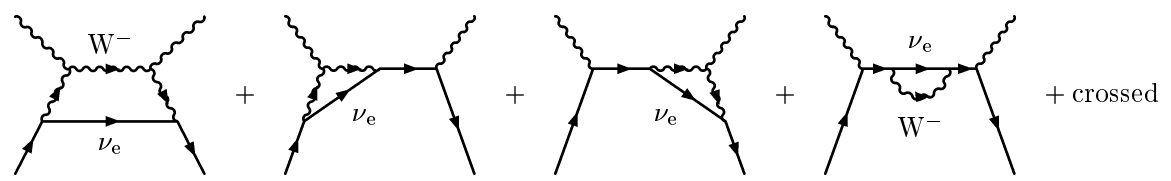

(d)

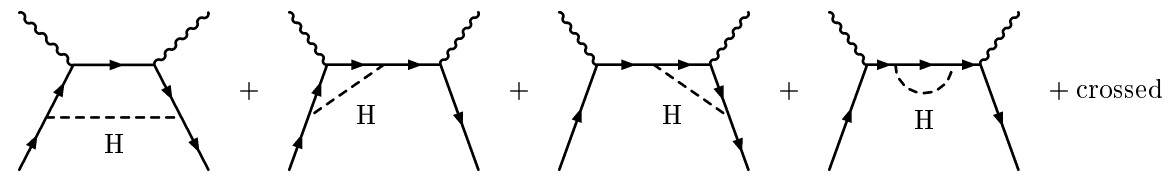

(e)

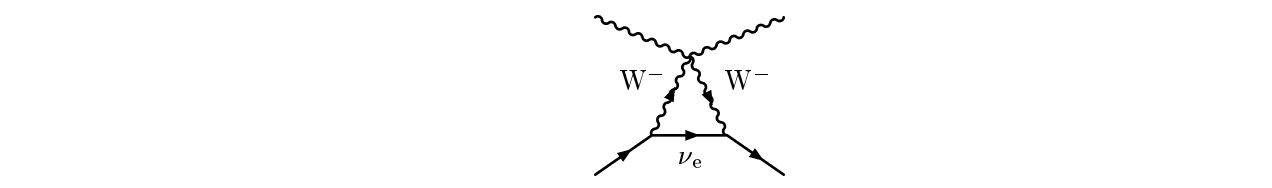

(f)

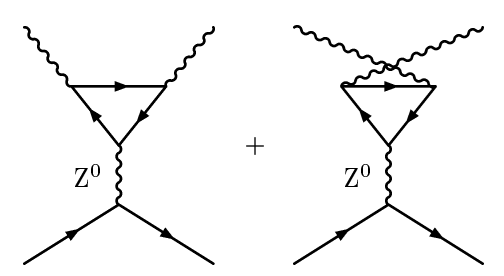

$(\mathrm{g})$
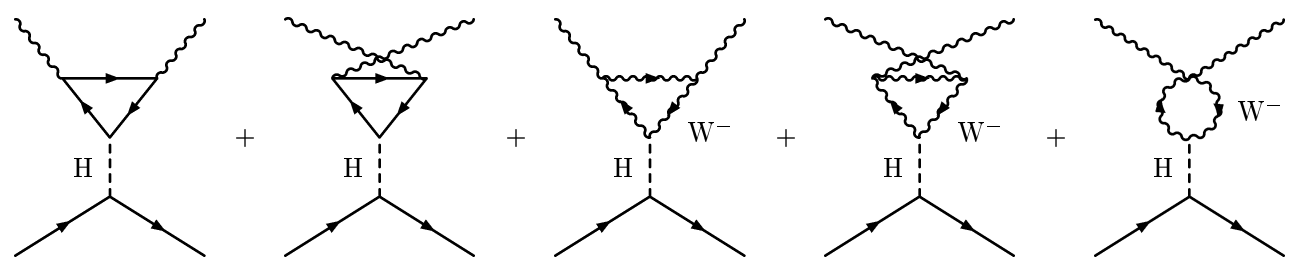

Figure 3: Feynman graphs contributing to the forward Compton amplitude of the $\mathcal{O}\left(\alpha^{2}\right)$ Weinberg-Salam model; solid lines represent $\mathrm{e}^{-}$and $\nu_{\mathrm{e}}$, wavy lines are the gauge bosons $\gamma, \mathrm{Z}^{0}$, and $\mathrm{W}^{ \pm}$, and a dashed line is the Higgs boson.
(a) tree graphs
(b) $\mathrm{e}^{-} \gamma$ and $\mathrm{e}^{-} \mathrm{Z}^{0}$ intermediate states
(c) $\nu_{\mathrm{e}} \mathrm{W}^{-}$intermediate state
(d) $\mathrm{e}^{-} \mathrm{H}$ intermediate state
(e) WW $\gamma \gamma$ contact graph
(f) Higgs exchange
(g) $\mathrm{Z}^{0}$ exchange

The graphs with crossed external photon lines are omitted in (b)-(d). Not shown are external line insertions such as vacuum polarization.

in contrast to the naive assumption (4.3). Re-inspecting now the derivation of the finite-momentum GDH sum rule presented in the previous section, we infer a modification given by

$$
0=\alpha \frac{G_{\mathrm{F}}}{\sqrt{2}}-\lim _{p^{0} \rightarrow \infty} \int_{0}^{\infty} \frac{\mathrm{d} \nu}{\nu} 8 \pi \operatorname{Im} f_{2}\left(\nu, \frac{m^{2} \nu^{2}}{\left(p^{0}\right)^{2}}\right) .
$$




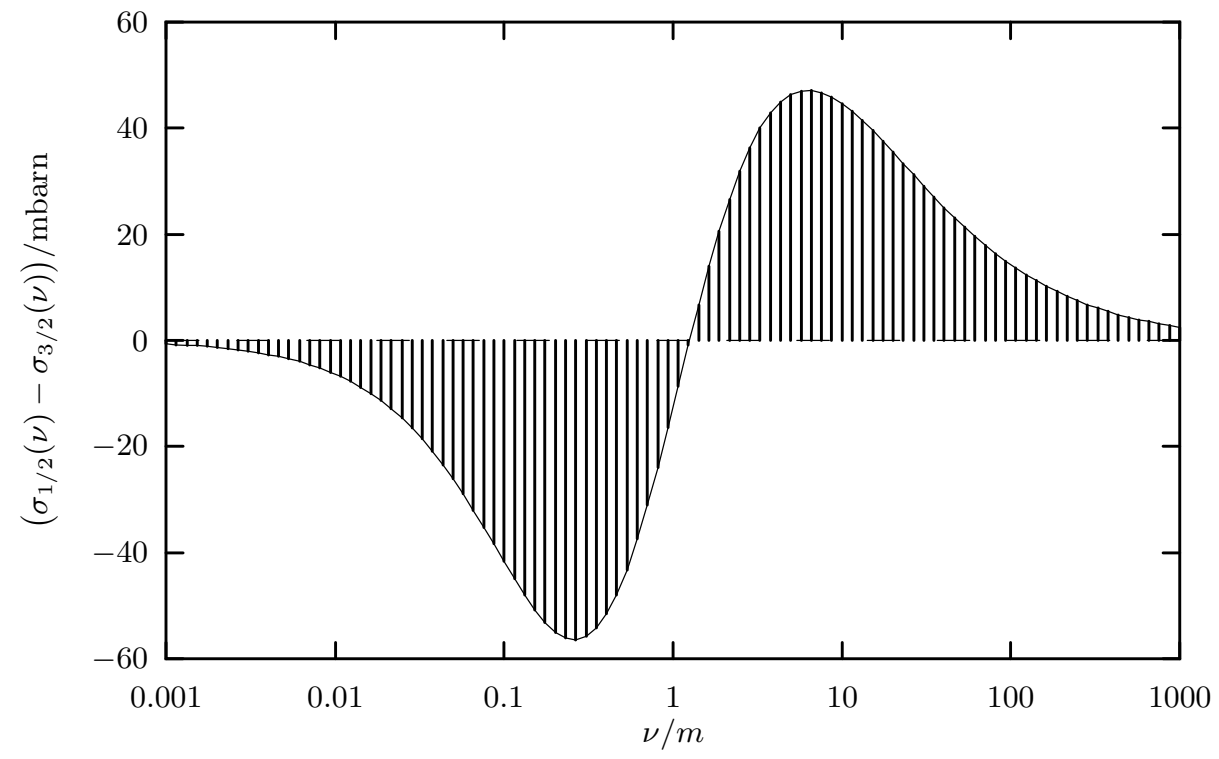

Figure 4: Polarized photoabsorption cross section of the electron in $\mathcal{O}\left(\alpha^{2}\right)$ QED. Since $\mathrm{d} \nu / \nu=\mathrm{d}(\ln \nu)$, the vanishing of (the QED contribution to) the integral (5.1) is reflected by the equality of the shaded areas.

Obviously, the GDH sum rule (5.1) would be violated if limit and integration were interchangeable. In the following we will show that due to the same Feynman graphs that gave rise to the anomalous commutator (5.4), dragging the limit inside the integral results in a second modification that cancels the first one. This means that the naive infinite-momentum limit is illegitimate here!

To prove this assertion, we calculated the $\mathrm{Z}^{0}$-exchange contribution to the amplitude $f_{2}\left(\nu, q^{2}\right)$. Some details are given in the appendix. The result is independent of the photon energy $\nu$,

$$
f_{2}^{(\mathrm{Z})}\left(\nu, q^{2}\right)=\frac{\alpha}{4 \pi^{2}} \frac{G_{\mathrm{F}}}{\sqrt{2}} f\left(q^{2}\right),
$$

where the function $f\left(q^{2}\right)$ can be given explicitely. As expected, it exhibits a branch-point singularity at the two-electron threshold $q^{2}=4 m^{2}$. Below this threshold one has

$$
\begin{aligned}
& \operatorname{Re} f\left(q^{2}\right)=\frac{4 m^{2}}{\sqrt{\left(4 m^{2}-q^{2}\right) q^{2}}} \operatorname{arccot} \sqrt{\frac{4 m^{2}}{q^{2}}-1}-1, \\
& \operatorname{Im} f\left(q^{2}\right)=0
\end{aligned}
$$

while for $q^{2}>4 m^{2}$,

$$
\begin{aligned}
& \operatorname{Re} f\left(q^{2}\right)=\frac{4 m^{2}}{\sqrt{\left(q^{2}-4 m^{2}\right) q^{2}}} \operatorname{artanh} \sqrt{1-\frac{4 m^{2}}{q^{2}}}-1, \\
& \operatorname{Im} f\left(q^{2}\right)=\frac{2 \pi m^{2}}{\sqrt{\left(q^{2}-4 m^{2}\right) q^{2}}} .
\end{aligned}
$$

This function is depicted in Fig. 5. For real Compton scattering, $q^{2}=0$, there is no contribution,

$$
f_{2}^{(\mathrm{Z})}(\nu, 0)=0,
$$

and hence

$$
\int_{0}^{\infty} \frac{\mathrm{d} \nu}{\nu} \operatorname{Im} f_{2}^{(\mathrm{Z})}(\nu, 0)=0 .
$$




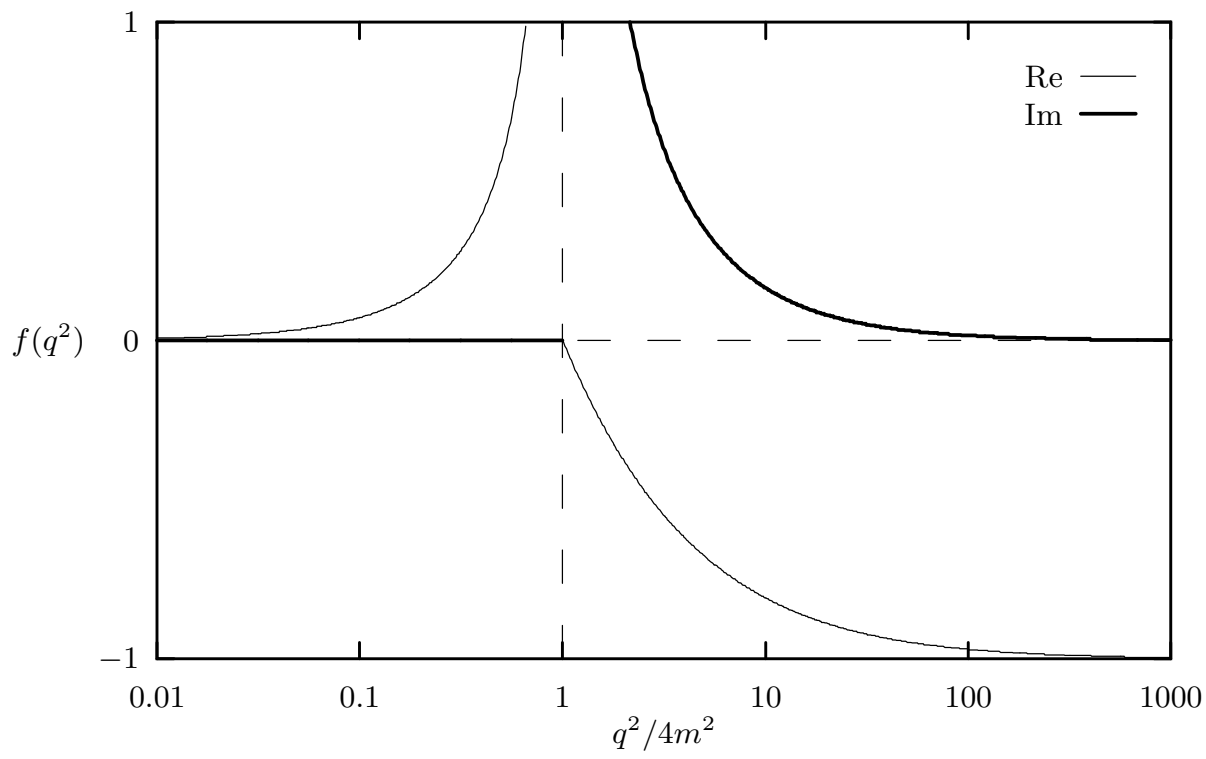

Figure 5: Real part (light line) and imaginary part (heavy line) of the function $f\left(q^{2}\right)$ occuring in the $\mathrm{Z}^{0}$-exchange contribution $f_{2}^{(\mathrm{Z})}\left(\nu, q^{2}\right) \propto f\left(q^{2}\right)$ to the virtual forward Compton amplitude of the $\mathcal{O}\left(\alpha^{2}\right)$ Weinberg-Salam model.

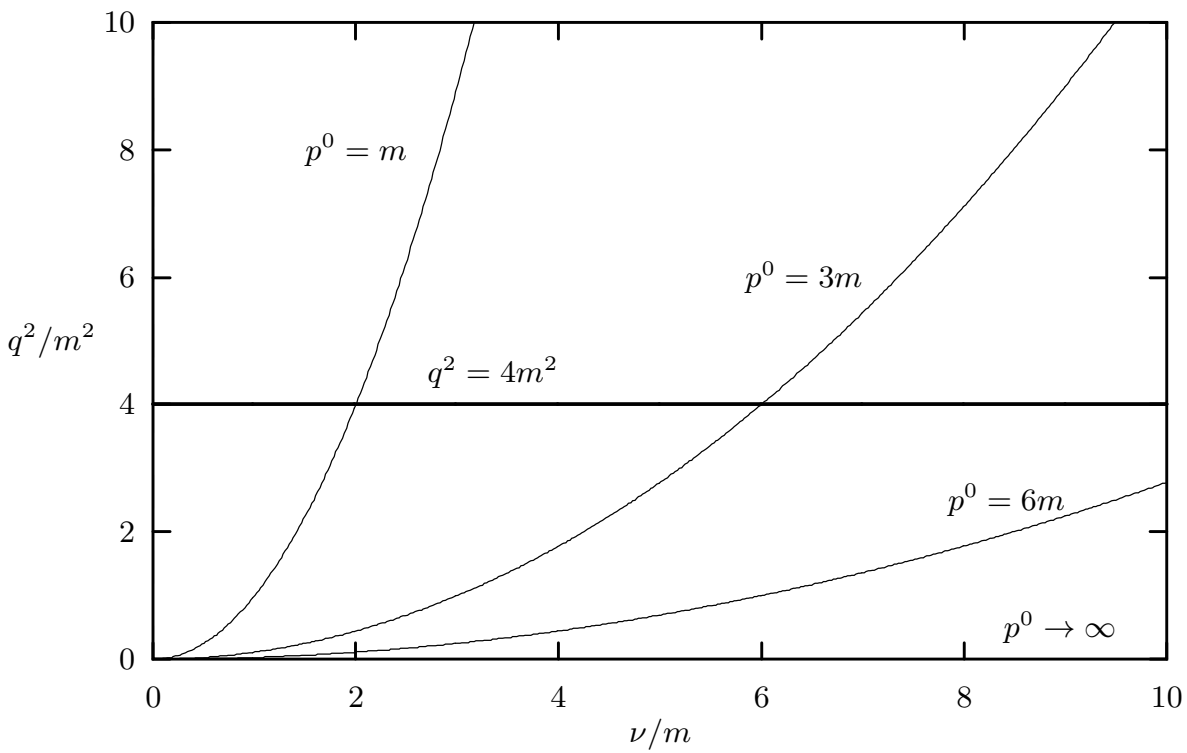

Figure 6: The integration path of the finite-momentum GDH sum rule of the $\mathcal{O}\left(\alpha^{2}\right)$ Weinberg-Salam model for electron energy $p^{0}=m, p^{0}=3 m$, and $p^{0}=6 m$. For any finite value of $p^{0}$ the integration passes the two-electron threshold $q^{2}=4 m^{2}$, picking up the constant on the right-hand side of Eq. (5.13).

However, for timelike photon virtualities above the two-electron threshold, one has a non-vanishing imaginary part. We infer from eqs. (5.6) and (5.10),

$$
\int_{0}^{\infty} \frac{\mathrm{d} \nu}{\nu} 8 \pi \operatorname{Im} f_{2}^{(\mathrm{Z})}\left(\nu, \frac{m^{2} \nu^{2}}{\left(p^{0}\right)^{2}}\right)=\alpha \frac{G_{\mathrm{F}}}{\sqrt{2}},
$$

which is independent of the electron energy $p^{0}$. As can be seen in Fig. 6, the contour of the integration in Eq. (5.13) passes the singularity line at $q^{2}=4 m^{2}$ for any finite value of $p^{0}$.

The crucial observation now is that interchanging the $\nu$ integration and the $p^{0} \rightarrow \infty$ limit 
leads to an additive constant

$$
\left(\lim _{p^{0} \rightarrow \infty} \int_{0}^{\infty} \frac{\mathrm{d} \nu}{\nu}-\int_{0}^{\infty} \frac{\mathrm{d} \nu}{\nu} \lim _{p^{0} \rightarrow \infty}\right) 8 \pi \operatorname{Im} f_{2}\left(\nu, \frac{m^{2} \nu^{2}}{\left(p^{0}\right)^{2}}\right)=\alpha \frac{G_{\mathrm{F}}}{\sqrt{2}},
$$

due to the $\mathrm{Z}^{0}$-exchange contributions 5.12 and $(5.13)$. Combined with the finite-momentum GDH sum rule (5.5), this leads back to the undisturbed GDH sum rule (5.1).

We remark that if quarks are included into the model, then the customary effect of anomaly cancellation removes the modification of the charge-density commutator as well as the one due to the infinite-momentum limit.

\section{Summary and conclusion}

We presented a derivation of the GDH sum rule from the equal-times commutator of electric charge densities. Our derivation exhibits the infinite-momentum limit as its last step. The finitemomentum GDH sum rule (4.8) gives the form that the sum rule takes without performing the infinite-momentum limit. We emphasized the fact that in principle, taking the infinite-momentum limit could give rise to a modification of the GDH sum rule, and that current algebra alone cannot tell whether such a modification is present or not.

To get a feeling for what can happen when taking the infinite-momentum limit, we considered virtual Compton scattering off the electron within the Weinberg-Salam model of weak interactions to order $\alpha^{2}$. We found that in this model the infinite-momentum limit does indeed give rise to a certain finite modification of the GDH sum rule, which comes together with (and is cancelled by) another modification due to an anomalous charge-density commutator.

The coincidence of these modifications leads us to the conclusion that any proposal of a modification of the GDH sum rule suggested from an anomalous charge-density commutator that has no regard to the legitimacy of the infinite-momentum limit (such as Ref. [10]) is to be seriously doubted!

\section{A $\quad Z^{0}$ exchange}

This appendix shall describe the calculation of the dipole-moment commutator matrix element (5.4) from the BJL formula (5.2), originating, to order $\alpha^{2}$, from the $\mathrm{Z}^{0}$-exchange graphs of Fig. 3(f). Also, the calculation of the contribution (5.6) of these graphs to the forward amplitude $f_{2}\left(\nu, q^{2}\right)$ is illustrated. The relevant Feynman integrals have been worked out by Rosenberg [26] and were discussed further by Adler 24.

We are concerned with the (generally non-forward) Compton amplitude

$$
e^{2} T^{\mu \nu}\left(p, p^{\prime}, q^{\prime}, s, s^{\prime}\right)=i e^{2} \int \mathrm{d}^{4} x e^{i q^{\prime} \cdot x}\left\langle p^{\prime}, s^{\prime}\left|\mathrm{T} J^{\mu}(x) J^{\nu}(0)\right| p, s\right\rangle .
$$

The contribution from the $\mathrm{Z}^{0}$-exchange graphs reads

$$
T_{(\mathrm{Z})}^{\mu \nu}=-\frac{M_{\mathrm{Z}}^{2} G_{\mathrm{F}}}{2 \sqrt{2}} R^{\mu \nu \rho}\left(q, q^{\prime}\right) \frac{-g_{\rho \sigma}+\left(p^{\prime}-p\right)_{\rho}\left(p^{\prime}-p\right)_{\sigma} / M_{\mathrm{Z}}^{2}}{\left(p^{\prime}-p\right)^{2}-M_{\mathrm{Z}}^{2}} \bar{u}\left(p^{\prime}, s^{\prime}\right) \gamma^{\sigma} \gamma_{5} u(p, s),
$$

with

$$
R^{\mu \nu \rho}=\int \frac{\mathrm{d}^{4} k}{(2 \pi)^{4}} \operatorname{Tr}\left(\gamma^{\mu} \frac{i}{\not k-m+i \epsilon} \gamma^{\nu} \frac{i}{\not k-\not k-m+i \epsilon} \gamma^{\rho} \gamma_{5} \frac{i}{\not k-\not q^{\prime}-m+i \epsilon}\right)+\left\{\begin{array}{c}
\mu \leftrightarrow \nu \\
q \leftrightarrow-q^{\prime}
\end{array}\right\} .
$$

The constant $M_{\mathrm{Z}}^{2} G_{\mathrm{F}} / 2 \sqrt{2}$ is due to the coupling of the $\mathrm{Z}^{0}$ to the electron lines, $m$ and $M_{\mathrm{Z}}$ are the masses of $\mathrm{e}^{-}$and $\mathrm{Z}^{0}$, respectively. The momentum four-vector $q$ of the incoming photon is fixed by $p+q=p^{\prime}+q^{\prime}$. This has to be kept in mind when the $q^{\prime 0} \rightarrow \infty$ limit is taken.

The triangle loop integral (A.3) can be cast into the form

$$
\begin{aligned}
R^{\mu \nu \rho}= & \epsilon^{\mu \nu \rho \alpha} q_{\alpha} R_{1}+\epsilon^{\nu \rho \alpha \beta} q_{\alpha}^{\prime} q_{\beta}\left(q^{\mu} R_{2}+q^{\prime \mu} R_{3}\right) \\
& +\epsilon^{\mu \nu \rho \alpha} q_{\alpha}^{\prime} R_{1}^{\prime}+\epsilon^{\mu \rho \alpha \beta} q_{\alpha}^{\prime} q_{\beta}\left(q^{\prime \nu} R_{2}^{\prime}+q^{\nu} R_{3}^{\prime}\right),
\end{aligned}
$$


where $R_{1,2,3}$ are functions of the Lorentz invariants $q^{2}, q^{\prime 2}$, and $q \cdot q^{\prime}$. From crossing symmetry, the primed quantities are given by $R_{1,2,3}^{\prime}\left(q^{2}, q^{\prime 2}, q \cdot q^{\prime}\right) \equiv R_{1,2,3}\left(q^{\prime 2}, q^{2}, q \cdot q^{\prime}\right)$. Gauge invariance $q_{\nu} T_{(\mathrm{Z})}^{\mu \nu}=0, q_{\mu}^{\prime} T_{(\mathrm{Z})}^{\mu \nu}=0$, imposes the condition

$$
R_{1}+q \cdot q^{\prime} R_{2}+q^{\prime 2} R_{3}=R_{1}^{\prime}+q \cdot q^{\prime} R_{2}^{\prime}+q^{2} R_{3}^{\prime}=0 .
$$

The crucial point of Ref. [26] is the observation that the functions $R_{2,3}$ are finite, while the formally divergent function $R_{1}$ can be fixed by the gauge-invariance condition (A.5). The result is given by the Feynman-parameter integrals

$$
R_{1,2,3}^{(\prime)}\left(q, q^{\prime}\right)=\frac{i}{\pi^{2}} \int_{0}^{1} \mathrm{~d} x \int_{0}^{1-x} \mathrm{~d} y \frac{N_{1,2,3}^{(\prime)}}{x(1-x) q^{2}+y(1-y) q^{\prime 2}-2 x y q \cdot q^{\prime}-m^{2}+i \epsilon},
$$

with the numerators

$$
\begin{aligned}
& N_{1}=x(1-x) q^{\prime 2}-x y q \cdot q^{\prime}, \quad N_{2}=x y, \quad N_{3}=-x(1-x), \\
& N_{1}^{\prime}=y(1-y) q^{2}-x y q \cdot q^{\prime}, \quad N_{2}^{\prime}=x y, \quad N_{3}^{\prime}=-y(1-y) .
\end{aligned}
$$

\section{A.1 Anomalous commutator}

To compute the matrix element of the dipole-moment commutator, we need the $q^{\prime 0} \rightarrow \infty$ limit of the time-time component $T_{(\mathrm{Z})}^{00}$. We have, from Eq. (A.4),

$$
\lim _{q^{\prime 0} \rightarrow \infty} q^{\prime 0} R^{00 \rho}=\epsilon^{0 \rho \alpha \beta} q_{\alpha}^{\prime} q_{\beta} \lim _{q^{\prime 0} \rightarrow \infty}\left(\left(q^{\prime 0}\right)^{2}\left(R_{2}+R_{2}^{\prime}+R_{3}+R_{3}^{\prime}\right)\right) .
$$

Carrying out the $q^{\prime 0} \rightarrow \infty$ limit in the explicite formulae (A.6) and (A.7), this reduces to

$$
\lim _{q^{\prime 0} \rightarrow \infty} q^{\prime 0} R^{00 \rho}=-\frac{i}{2 \pi^{2}} \epsilon^{0 \rho \alpha \beta} q_{\alpha}^{\prime} q_{\beta} .
$$

Inserting this into Eq. (A.2) and going back to the BJL formula (5.2), it is a trivial matter to work out the matrix element of the charge-density commutator,

$$
\begin{aligned}
& \left\langle p^{\prime}, \frac{1}{2}\left|\left[J^{0}(\boldsymbol{x}, 0), J^{0}(0)\right]\right| p, \frac{1}{2}\right\rangle= \\
& \quad-\epsilon^{i j k} \frac{M_{\mathrm{Z}}^{2} G_{\mathrm{F}}}{4 \pi^{2} \sqrt{2}} \frac{\left(p^{\prime}-p\right)^{i} \bar{u}\left(p^{\prime}, \frac{1}{2}\right) \gamma^{j} \gamma_{5} u\left(p, \frac{1}{2}\right) \nabla^{k} \delta^{3}(\boldsymbol{x})}{\left(p^{\prime}-p\right)^{2}-M_{\mathrm{Z}}^{2}} .
\end{aligned}
$$

The matrix element (5.4) of the dipole-moment commutator is now obtained by using translational invariance and the properties of the helicity spinor $u\left(p, \frac{1}{2}\right)$.

\section{A.2 Infinite-momentum limit}

On the other hand, we want to compute the $\mathrm{Z}^{0}$-exchange contribution to the forward Compton amplitude $f_{2}\left(\nu, q^{2}\right)$. To this end, we have to specialize eqs. (A.2) and (A.4) to the case $p=p^{\prime}$, $q=q^{\prime}, s=s^{\prime}$, giving

$$
T_{(\mathrm{Z})}^{\mu \nu}(p, q, s)=m \frac{G_{\mathrm{F}}}{\sqrt{2}} \epsilon^{\mu \nu \rho \sigma} q_{\rho} s_{\sigma}\left(R_{1}+R_{1}^{\prime}\right) .
$$

Recalling the invariant decomposition (2.2), we notice that $\mathrm{Z}^{0}$ exchange contributes to $A_{1}\left(\nu, q^{2}\right)$ only,

$$
\begin{aligned}
A_{1}^{(\mathrm{Z})}\left(\nu, q^{2}\right) & =-\frac{m^{2}}{\pi^{2}} \frac{G_{\mathrm{F}}}{\sqrt{2}} \int_{0}^{1} \mathrm{~d} x \int_{0}^{1-x} \mathrm{~d} y \frac{(x+y)(1-x-y) q^{2}}{(x+y)(1-x-y) q^{2}-m^{2}+i \epsilon} \\
& =\frac{m^{2}}{2 \pi^{2}} \frac{G_{\mathrm{F}}}{\sqrt{2}} f\left(q^{2}\right),
\end{aligned}
$$


where

$$
f\left(q^{2}\right)=-2 \int_{0}^{1} \mathrm{~d} z \frac{z^{2}(1-z) q^{2}}{z(1-z) q^{2}-m^{2}+i \epsilon} .
$$

This implies Eq. (5.6) via relation (2.3). The $z$ integration can be performed explicitely, giving formulae (5.7)- 5.10$)$.

Finally, it is perhaps worth noting that the exact cancellation of the two modifications of the GDH sum rule can be traced back to the gauge-invariance condition (A.5), which relates the function $R_{1}$ occuring in Eq. A.11) to the functions $R_{2,3}$ of Eq. (A.9).

\section{References}

[1] S. B. Gerasimov, Yad. Fiz. 2, 598 (1965), [Sov. J. Nucl. Phys. 2, 430 (1966)].

[2] S. D. Drell and A. C. Hearn, Phys. Rev. Lett. 16, 908 (1966).

[3] G. C. Fox and D. Z. Freedman, Phys. Rev. 182, 1628 (1969).

[4] I. Karliner, Phys. Rev. D 7, 2717 (1973).

[5] R. L. Workman and R. A. Arndt, Phys. Rev. D 45, 1789 (1992).

[6] A. M. Sandorfi, C. S. Whisnant, and M. Khandaker, Phys. Rev. D 50, R6681 (1994).

[7] T. Coersmeier, Diploma Thesis (Bonn, 1993).

[8] K. Kawarabayashi and M. Suzuki, Phys. Rev. 152, 1383 (1966).

[9] A. Khare, Prog. Theor. Phys. 53, 1798 (1975).

[10] L. N. Chang, Y. Liang, and R. L. Workman, Phys. Lett. B 329, 514 (1994).

[11] A. H. Mueller and T. L. Trueman, Phys. Rev. 160, 1306 (1967).

[12] B. L. Ioffe, V. A. Khoze, and L. N. Lipatov, Hard Processes (North Holland, Amsterdam, 1984).

[13] H. D. I. Abarbanel and M. L. Goldberger, Phys. Rev. 165, 1594 (1968).

[14] M. Hosoda and K. Yamamoto, Prog. Theor. Phys. 35, 425 (1966).

[15] K. Kawarabayashi and M. Suzuki, Phys. Rev. 150, 1181 (1966).

[16] G. Altarelli, N. Cabibbo, and L. Maiani, Phys. Lett. B 40, 415 (1972).

[17] C. Itzykson and J.-B. Zuber, Quantum Field Theory (McGraw-Hill, New York, 1980).

[18] F. E. Low, Phys. Rev. 96, 1428 (1954).

[19] M. Gell-Mann and M. L. Goldberger, Phys. Rev. 96, 1433 (1954).

[20] T. Pradhan and A. V. Khare, Phys. Rev. 5, 496 (1972).

[21] S. J. Brodsky and I. Schmidt, Phys. Lett. B 351, 344 (1995).

[22] J. D. Bjorken, Phys. Rev. 148, 1467 (1966).

[23] K. Johnson and F. E. Low, Supp. Prog. Theor. Phys. 37/38, 74 (1966).

[24] S. L. Adler, Phys. Rev. 177, 2426 (1969).

[25] J. S. Bell and R. Jackiw, Nuovo Cim. 60A, 47 (1969).

[26] L. Rosenberg, Phys. Rev. 129, 2786 (1963). 